\title{
Predictive Factors for Anastomotic Leakage after Simultaneous Resection of Synchronous Colorectal Liver Metastasis
}

\author{
Kentaro Nakajima • Shinichiro Takahashi • \\ Norio Saito • Masahito Kotaka • Masaru Konishi • \\ Naoto Gotohda • Yuichiro Kato • Taira Kinoshita
}

Received: 15 September 2011 / Accepted: 11 November 2011 / Published online: 29 November 2011

(C) 2011 The Author(s). This article is published with open access at Springerlink.com

\begin{abstract}
Background The optimal surgical strategy for resectable, synchronous, colorectal liver metastases remains unclear. The objective of this study was to determine which patients could benefit from staged resections instead of simultaneous resection by identifying predictive factors for postoperative morbidity and anastomotic leakage after simultaneous resection of synchronous, colorectal liver metastases and the primary colorectal tumor.

Methods This study involved 86 patients with synchronous colorectal liver metastases who underwent simultaneous resection of the primary colorectal tumor and the hepatic tumor. Postoperative mortality, morbidity, and other surgical outcomes, including survival and hospitalization, were assessed. Predictive factors for postoperative morbidity and for anastomotic leakage were evaluated.

Results Postoperative morbidity and anastomotic leakage were found in 55 (64\%) and 18 (21\%) patients. Predictive factors for postoperative morbidity and for anastomotic leakage were intraoperative blood loss and operation time $>8 \mathrm{~h}$, respectively. The overall 5-year survival rate was $45 \%$.

Conclusions The frequency of morbidity and that of anastomotic leakage seemed to be high after simultaneous resection for synchronous colorectal liver metastases, especially when intraoperative blood loss or operation time increased greatly. Staged resections should be considered in cases in which excessive surgical stress from simultaneous resection of synchronous colorectal liver metastases would be expected.
\end{abstract}

Keywords Colorectal cancer $\cdot$ Hepatic metastasis $\cdot$ Liver metastasis $\cdot$ Morbidity $\cdot$ Anastomotic leakage

\footnotetext{
K. Nakajima $\cdot$ N. Saito

Department of Colorectal Surgery,

National Cancer Center Hospital East,

Kashiwa, Chiba, Japan

S. Takahashi $(\bowtie) \cdot$ M. Konishi $\cdot$ N. Gotohda $\cdot$ Y. Kato $•$

T. Kinoshita

Department of Hepato-biliary Pancreatic Surgery,

National Cancer Center Hospital East,

6-5-1 Kashiwanoha,

Kashiwa 277-8577, Chiba, Japan

e-mail: shtakaha@east.ncc.go.jp

M. Kotaka

Department of Surgery, Sano Hospital,

Kobe, Hyogo, Japan
}

\section{Introduction}

For patients with synchronous colorectal liver metastases (SCLM), hepatic resection is considered the best treatment, with reported 5-year survival rates between $23 \%$ and $37 \% .^{1-4}$ Resections of both the primary colorectal lesion and the hepatic metastases are needed for patients with SCLM when they are resectable. However, the optimal surgical strategy for resectable SCLM still remains controversial.

From the perspectives of less operation with less mental stress and simplifying perioperative treatment, simultaneous resection of the primary colorectal and liver tumors is a favorable strategy for patients with SCLM ${ }^{5-8}$ However, several papers reported that the morbidity rate after simultaneous resection of primary and liver tumors was high because of greater surgical stress and a longer 
operation time than for single-organ surgery. Staged resection with initial operation for the primary lesion followed by resection of hepatic tumors is regarded as an alternative strategy to avoid excessive surgical stress for patients with SCLM, though the efficacy of this strategy and the patients who could benefit from this strategy are unknown. ${ }^{4-6,9,10}$

Thus, this study was conducted to determine which patients could benefit from staged resections instead of simultaneous resection by identifying predictive factors for postoperative morbidity and anastomotic leakage after simultaneous resection of SCLM.

\section{Patients and Methods}

\section{Patient Population}

The medical records of all consecutive patients who underwent liver resections for colorectal liver metastases from January 1992 to January 2004 at our institution were analyzed retrospectively, with institutional review board approval. Eighty-six patients had SCLM. During this period, all SCLM patients received simultaneous resection of primary colorectal and hepatic tumors irrespective of the patient's or the tumor's characteristics. Lateral lymph node dissection was routinely performed in patients with advanced lower rectal cancer. All 86 patients underwent contrast enhanced computed tomography (CT) of the chest, abdomen, and pelvis, as well as hepatic MRI, preoperatively.

As a control, the morbidity of 167 patients who underwent hepatectomy for metachronous liver metastasis from colorectal cancer from January 1992 to January 2004 and that of 1,728 patients who underwent only resection for colorectal cancer with colorectal reconstruction during the same period were also reviewed. Of the 1,728 colorectal cancer patients, 1,319 had colon cancer and 409 had rectal cancer.

\section{Postoperative Morbidity}

Incidences of the following postoperative complications were analyzed: anastomotic leak, rectovaginal fistula, intraperitoneal or pelvic abscess, wound infection, wound dehiscence, ileus, enteroparesis, postoperative delirium, urinary tract infection, dysuria, empyema thoracis, pleural effusion, atelectasis, cholecystitis, perihepatic or subphrenic abscess, bile leak, liver failure, and others. Anastomotic leakage was defined as follows: peritonitis and a dehiscence in the anastomosis, discharge of pus from the anus, vaginal fistula, or feces from the abdominal drain. Leakage was confirmed by $\mathrm{CT}$ scan, contrast enema, re-operation, or digital rectal examination. All complications were graded according to the classification proposed by Clavien et al. ${ }^{11}$ Postoperative mortality was defined to include any death during postoperative hospitalization or within 30 days.

\section{Assessment of Predictive Factors for Postoperative Morbidity}

Correlations between postoperative morbidity and the following patient, tumor, and surgical factors were analyzed: age, sex, body mass index (BMI), preoperative comorbidity, site of primary tumor, intestinal obstruction by tumor, size of primary tumor, differentiation of tumor, distribution of hepatic tumors, number of hepatic tumors, hepatic tumor size, operative methods, operation time, intraoperative blood loss, and blood transfusion.

\section{Survival}

Patients were followed regularly at 3-month intervals with blood testing and CT. Survival and follow-up were calculated from the time of the operation to the date of death or last available follow-up. The survivors' median follow-up time after surgery was 73 months.

\section{Statistical Analysis}

Statistical comparisons of baseline data were performed using the chi-square test. Continuous variables were compared with the independent $t$ test. Multivariate analyses to evaluate the independent predictive factors for postoperative complications or anastomotic leakage were done by multiple logistic regression analysis. The survival rate was calculated by the Kaplan-Meier method. ${ }^{12}$ A difference was considered significant when $p$ was less than 0.05 .

\section{Results}

Patients and Operative Details

From 1992 to 2004, 86 patients were treated with simultaneous resection of primary and hepatic tumors for SCLM. There were 37 female and 49 male patients, with a median age of 59 years (range, 40 to 85 years). The site of the primary tumor was colon in 48 and rectum in 38. The primary tumor was staged as $\mathrm{T} 3$ in $54(63 \%)$ and $\mathrm{T} 4$ in $32(37 \%)$ according to the TNM classification. Metastatic lymph nodes were found in 65 patients $(76 \%)$. The mean diameter of the primary tumor was $55 \mathrm{~mm}$ (range, 26-140 $\mathrm{mm}$ ).

Liver metastases were solitary in 29 patients and multiple in 57 patients. In 47 patients (55\%), the hepatic 
tumor showed a unilobar distribution, while a bilobar tumor distribution was observed in $39(45 \%)$. The mean diameter of the hepatic tumor was about $43 \mathrm{~mm}$ (range, 5-200 mm). The mean resected liver volume was $380 \mathrm{~g}$ (range, 10-1,660 g).

The operation for primary colorectal cancer was right (hemi) colectomy in 17 patients, transverse colectomy in 1 , left (hemi) colectomy in 4 , sigmoidectomy in 24 , high anterior resection in 7 , low anterior resection in 20 , very low anterior resection in 6, inter-sphincteric resection in 2 , Hartmann's operation in 1, and abdomino-perineal resection in 4 (Table 4). A diverting stoma to prevent anastomotic leakage was made in $22(26 \%)$ patients at the surgeon's discretion, and lateral lymph node dissection was performed in $20(23 \%)$. In terms of liver tumor resection, lobectomy was performed in 11 patients, segmentectomy in 22 , bisegmentectomy in 1 , trisegmentectomy in 2, subsegmentectomy in 3, and partial resection in 47.

Adjuvant therapy was given to only 17 patients (19.8\%) because adjuvant chemotherapy for colorectal cancer in stage III or more was performed since January 2003. Neoadjuvant chemoradiation targeting for rectal cancer was given to three patients $(3.5 \%)$.

\section{Morbidity}

No patients died within 30 days of the operation, but 55 (64\%) patients developed complications (Table 1). Eighteen patients $(21 \%)$ experienced leakage, of whom 6 needed urgent re-operation with ileostomy and drainage of an intraabdominal collection caused by leakage. Postoperative bleeding, wound dehiscence, and ileus were the reasons for the three other re-operation cases. The most frequent complication was wound infection.

The morbidity rate of the 167 patients who underwent hepatectomy for metachronous colorectal liver metastasis during the same period was $19.8 \%$, and that of 1,728 patients who underwent only resection for colorectal cancer was $32.1 \%$. Anastomotic leakage occurred in $123(7.1 \%)$ of the aforementioned 1,728 patients.

Factors Affecting Complications, Especially Anastomotic Leakage

Postoperative complications were significantly correlated with presence of diverting stoma $(p<0.01)$, duration of operation greater than $8 \mathrm{~h}(p<0.01)$, amount of intraoperative blood loss $(p<0.01)$, and intraoperative blood transfusion $(p<0.01)$. The aforementioned factors were entered into multivariate analysis. Only a greater amount of blood loss had a predictive value for increased occurrence of postoperative complications.

Then, the correlations between anastomotic leakage and clinicopathological factors were examined to identify risk factors for anastomotic leakage after simultaneous resection for SCLM. Patients who underwent abdomino-perineal
Table 1 Postoperative complications after simultaneous resection for SCLM according to Clavien grade

\begin{tabular}{|c|c|c|c|c|c|c|}
\hline Complications & No. of patients & Gr I & Gr II & Gr IIIa & Gr IIIb & Gr IVa \\
\hline \multicolumn{7}{|l|}{ Colon and rectum } \\
\hline Anastomotic leakage & $18(21 \%)$ & & 12 & & 6 & \\
\hline Intrapelvic abscess & $6(7 \%)$ & 1 & 4 & & 1 & \\
\hline Intraperitoneal abscess & $5(6 \%)$ & 1 & 0 & 3 & 1 & \\
\hline Rectovaginal fistula & $4(5 \%)$ & & 1 & & 3 & \\
\hline \multicolumn{7}{|l|}{ Liver } \\
\hline Bile leakage & $7(8 \%)$ & 6 & 1 & & & \\
\hline Hepatic abscess & $7(8 \%)$ & & 5 & 1 & 1 & \\
\hline Liver failure & $3(3 \%)$ & 1 & 1 & & & 1 \\
\hline Postoperative bleeding & $1(1 \%)$ & & & & 1 & \\
\hline \multicolumn{7}{|l|}{ Other organs } \\
\hline Wound infection & $25(29 \%)$ & 23 & 2 & & & \\
\hline Pleural effusion & $12(14 \%)$ & 1 & & 11 & & \\
\hline Wound dehiscence & $6(7 \%)$ & 3 & 2 & & 1 & \\
\hline Enteroparesis & $5(6 \%)$ & 5 & & & & \\
\hline Postoperative delirium & $4(5 \%)$ & 1 & 3 & & & \\
\hline Dysuria & $4(5 \%)$ & & 4 & & & \\
\hline Urinary tract infection & $3(3 \%)$ & & 3 & & & \\
\hline Pneumonia & $2(2 \%)$ & & 2 & & & \\
\hline Others & $7(8 \%)$ & 1 & 4 & & 2 & \\
\hline
\end{tabular}


resection $(n=4)$ or Hartmann's operation $(n=1)$ were excluded from the analysis. Anastomotic leakage was significantly correlated with lateral lymph node dissection $(p<0.01)$, primary site of rectum $(p=0.01)$, duration of operation greater than $8 \mathrm{~h}(p<0.01)$, and amount of intraoperative blood loss $(p=0.02)$. Neither serum levels of TP and ALB, steroid usage, nor neoadjuvant therapy showed correlation with occurrence of anastomotic leakage (data not shown). Multivariate analyses revealed operation time greater than $8 \mathrm{~h}(p<0.01)$ as the only independent predictive factor for anastomotic leakage after simultaneous resection of SCLM (Table 2). Extent of hepatectomy, timing of anastomosis and hepatectomy, and usage of Pringle maneuver did not correlate with occurrence of complication or anastomotic leakage.

Table 3 showed the rates of complication $\geq$ IIIa and anastomotic leakage according to operative procedures of the primary and hepatic resections which were performed in the same patient. Complication $\geq$ IIIa and anastomotic leakage were more frequently observed in patients with rectal resection; however, extent of hepatectomy did not seem to affect occurrence of complication $\geq$ IIIa or anastomotic leakage.

Hospitalization was significantly longer in the 55 patients with postoperative morbidity ( 32.2 days) than in the 31 patients without postoperative morbidity (17.6 days) $(p<0.01)$. In addition, hospitalization was significantly longer in the 18 patients with anastomotic leakage (43.5 days) than in the 63 patients without anastomotic leakage (22.2 days) $(p<0.01)$.

\section{Survival}

The overall survival rate after simultaneous resection for SCLM of the 86 patients was $61 \%$ at 3 years and $45 \%$ at 5 years, with MST of 47 months.

\section{Discussion}

For patients with resectable SCLM, both primary tumor resection and hepatectomy for liver metastasis could lead to long-term survival, with a 5-year survival rate of $23-37 \%$. However, the optimal strategy, including surgical resection and perioperative treatment, remains controversial for resectable SCLM. In terms of surgical resection for SCLM, it has not been resolved whether simultaneous resection or staged resections would be preferable.

There are several rationales for simultaneous resection of SCLM. In simultaneous resection, the treatment strategy would become simpler. In the staged resections, a series of neoadjuvant chemotherapy or chemoradiotherapy, resection of primary tumor, chemotherapy between two operations, hepatectomy, and adjuvant chemotherapy could be the maximal total treatment for SCLM, while simultaneous resection could simplify and shorten the treatment schedule by eliminating one operation. Completion of the two resections and initiation of adjuvant chemotherapy occur earlier with simultaneous resection than with staged resections. Considering survival, comparable survival for simultaneous resection was shown in comparison with that for staged resections. ${ }^{13}$ Furthermore, simultaneous resection could relieve patients from a considerable degree of mental and physical stress and decrease total treatment cost by preventing a second resection for hepatic metastases. Recent advances in colorectal and hepatic surgery have enabled simultaneous resection to be performed more safely. Martin et al. reported the safety and efficacy of simultaneous resection. By avoiding a second laparotomy, the overall complication rate was reduced, and length of hospital stay was shortened, with no change in operative mortality. 7,8

However, at present, staged resections with initial resection of the primary tumor followed by hepatic resection have been frequently performed in patients with SCLM for several reasons. ${ }^{4,5,9,10}$ First, the perioperative risk of staged resections has been thought to be less than that of simultaneous resection. ${ }^{4,13,14}$ Sheele et al. reported 13 anastomotic leakages of 90 simultaneous procedures in their series, and two of them led to death. ${ }^{4}$ Thelen et al. proposed the criteria for simultaneous liver resection according to the age and extent of liver resection, because death after simultaneous liver resection $(n=4)$ occurred after major hepatectomies, and three of these four patients were 70 years of age or older. ${ }^{15}$ Second, staged resections might offer a chance to evaluate liver or extrahepatic metastases between the two operations. Lambert et al. reported that staged resections of synchronous hepatic metastases with an interval of 3 to 6 months might allow occult disease to become clinically detectable and could potentially identify patients for whom a hepatic resection would offer no survival benefit. ${ }^{10}$ Fujita recommended an interval resection to assess the metastatic status of the regional lymph nodes, because the presence of six or more lymph node metastases was an independent poor prognostic factor in patients with resected SCLM and a relative contraindication for hepatic resection. ${ }^{9}$ Some authors proposed chemotherapy between primary tumor resection and liver resection to select patients that could benefit from hepatectomy. ${ }^{13,16}$ Alternatively, a liver-first approach of doing liver resection first and primary resection second was newly proposed as a strategy for SCLM. ${ }^{17,18}$ The liver-first approach might avoid needless radical colorectal surgery by confirming curability of hepatic metastases first and also might increase resectability compared with the ordinary staged resections especially in patients with progressive hepatic metastases. 
Table 2 Correlation between anastomotic leakage and clinicopathological factors in patients who underwent simultaneous resection for SCLM

\begin{tabular}{|c|c|c|c|c|c|}
\hline \multirow{2}{*}{\multicolumn{2}{|c|}{$\overline{\text { Patient characteristics }}$}} & $\begin{array}{l}\text { Leakage }(-) \\
(n=63)\end{array}$ & $\begin{array}{l}\text { Leakage }(+) \\
(n=18)\end{array}$ & $\begin{array}{l}\text { Univariate analysis } \\
p \text { value }\end{array}$ & $\begin{array}{l}\text { Multivariate analysis } p \text { value, } \\
\text { RR }(95 \% \text { CI })\end{array}$ \\
\hline & & & & & \\
\hline \multicolumn{2}{|c|}{ Median age (range) (years) } & $59(40-85)$ & $59(41-73)$ & 0.81 & \\
\hline \multicolumn{2}{|c|}{ Male/female } & $33 / 30$ & $12 / 6$ & 0.42 & \\
\hline \multicolumn{2}{|c|}{ BMI (mean \pm SD) } & $21.9 \pm 2.9$ & $22.5 \pm 2.2$ & 0.44 & \\
\hline \multicolumn{6}{|c|}{ Preoperative comorbidity } \\
\hline \multicolumn{2}{|l|}{ Absent } & 44 & 12 & 0.78 & \\
\hline \multicolumn{2}{|l|}{ Present } & 19 & 6 & & \\
\hline \multicolumn{6}{|c|}{ Primary colorectal tumor } \\
\hline \multirow[t]{2}{*}{ Site } & Colon & 42 & 6 & 0.01 & N.S. \\
\hline & Rectum & 21 & 12 & & \\
\hline \multirow[t]{2}{*}{ Stenosis } & Absent & 56 & 0 & 0.34 & \\
\hline & Present & 7 & 18 & & \\
\hline \multicolumn{2}{|c|}{ Tumor size, mm } & 52.0 & 58.0 & 0.25 & \\
\hline \multirow[t]{2}{*}{ pT stage } & pT3 & 41 & 9 & 0.25 & \\
\hline & pT4 & 22 & 9 & & \\
\hline \multirow[t]{2}{*}{$\mathrm{pN}$ stage } & $\mathrm{pN} 0$ & 17 & 2 & 0.22 & \\
\hline & $\mathrm{pN}+$ & 46 & 16 & & \\
\hline \multirow[t]{2}{*}{ Histology } & Well, mod & 60 & 15 & 0.12 & \\
\hline & Poor & 3 & 3 & & \\
\hline \multicolumn{6}{|c|}{ Liver metastasis } \\
\hline \multirow[t]{2}{*}{ Distribution } & Unilobar & 38 & 9 & 0.43 & \\
\hline & Bilobar & 25 & 9 & & \\
\hline \multicolumn{2}{|c|}{ Number of tumors (range) } & $2.3(1-8)$ & $2.6(1-8)$ & 0.57 & \\
\hline \multicolumn{2}{|c|}{ Tumor size, mm } & 47 & 33 & 0.06 & \\
\hline \multicolumn{6}{|c|}{ Operative factors } \\
\hline \multicolumn{6}{|c|}{ Lateral lymph node dissection } \\
\hline \multicolumn{2}{|l|}{ Absent } & 55 & 10 & $<0.01$ & N.S. \\
\hline Present & & 8 & 8 & & \\
\hline Diverting st & & & & & \\
\hline Absent & & 48 & 11 & 0.24 & \\
\hline Present & & 15 & 7 & & \\
\hline Liver resect & & & & & \\
\hline Partial Hx, & omy & 51 & 16 & 0.72 & \\
\hline$\geq$ Lobectom & & 12 & 2 & & \\
\hline Timing of a & & & & & \\
\hline Colectomy & $\mathrm{sis} \rightarrow \mathrm{Hx}$ & 20 & 4 & 0.20 & \\
\hline Colectomy & stomosis & 7 & 5 & & \\
\hline $\mathrm{Hx} \rightarrow$ colect & tomosis & 36 & 9 & & \\
\hline Pringle man & & & & & \\
\hline Absent & & 10 & 1 & 0.44 & \\
\hline Present & & 53 & 17 & & \\
\hline Operation ti & & & & & \\
\hline$<8 \mathrm{~h}$ & & 53 & 8 & $<0.01$ & $<0.01,6.63(2.09-20.9)$ \\
\hline$\geq 8 \mathrm{~h}$ & & 10 & 10 & & \\
\hline Blood loss, & & $1,345(162-6,000)$ & $2,487(430-6,560)$ & 0.02 & N.S. \\
\hline Transfusion & & & & & \\
\hline Absent & & 39 & 9 & 0.37 & \\
\hline Present & & 24 & 9 & & \\
\hline Blood trans & & 343 & 1,212 & 0.05 & \\
\hline
\end{tabular}

$R R$ relative risk, $C I$ confidence interval, $H x$ hepatectomy, $N . S$. non-significant $(p>0.05)$ 
Table 3 Rates of complication $\geq$ Gr IIIa and anastomotic leakage according to the site of primary colorectal resection and extent of hepatectomy

\begin{tabular}{llrr}
\hline Primary colorectal resection & Hepatectomy & Complication $\geq$ Gr IIIa & Anastomotic leakage \\
\hline Colectomy & $<$ Lobectomy & $4 / 40(10 \%)$ & $5 / 39^{\mathrm{a}}(13 \%)$ \\
& $\geq$ Lobectomy & $0 / 7(0 \%)$ & $1 / 7(14 \%)$ \\
Rectal resection & $<$ Lobectomy & $11 / 32(34 \%)$ & $11 / 28^{\mathrm{b}}(39 \%)$ \\
& <Lobectomy & $2 / 7(29 \%)$ & $1 / 7(14 \%)$ \\
\hline
\end{tabular}

${ }^{a}$ One patient who underwent Hartmann's operation was excluded from the analysis

${ }^{\mathrm{b}}$ Four patients who underwent abdomino-perineal resection were excluded from the analysis

This study evaluated morbidity, especially anastomotic leakage, after simultaneous resection for SCLM in order to assess the safety of simultaneous resection. Anastomotic leakage is sometimes fatal and can cause a difficult situation with physical and mental discomfort or pain. The morbidity rate of patients who underwent simultaneous resection for SCLM seemed to be higher than that of patients with resected metachronous colorectal hepatic metastasis or that of patients who underwent only resection for colorectal primary cancer. Predictive factors for postoperative morbidity and for anastomotic leakage were intraoperative blood loss and operation time greater than $8 \mathrm{~h}$, respectively. The overall morbidity rate and the rate of anastomotic leakage were $91 \%$ and $50 \%$, respectively, in patients with operation time greater than $8 \mathrm{~h}$, and $54 \%$ and $13 \%$, respectively, in patients with operation time less than or equal to $8 \mathrm{~h}$. Blood loss and operation time usually represent the amount of surgical stress. Excessive surgical stress was possibly correlated with postoperative morbidity. Hospitalization of patients with complications was significantly longer than that of patients without complications. In particular, the average hospitalization of the 18 patients with anastomotic leakage was more than 43 days. Retrospective studies have also indicated that the occurrence of anastomotic leakage is associated with increased morbidity, mortality, and prolonged hospital stay. Additionally, anastomotic leakage may be associated with an increased risk of local recurrence. ${ }^{19}$

Various risk factors for anastomotic leakage have been analyzed by several investigators. Age, sex, obesity, level of anastomosis, smoking, blood transfusion, tumor diameter, preoperative (chemo) radiotherapy, physical status, obstruction, and coronary heart disease have been shown to be significant risk factors for leakage. ${ }^{20-24}$ In simultaneous resection for SCLM, not only the factors related to the tumor, the patient, or the colorectal operation, but factors related to the hepatectomy could affect the occurrence of anastomotic leakage. However, the extent of hepatic resection, sequence of colectomy, hepatectomy, anastomosis, use of the Pringle maneuver, and total time of the Pringle maneuver were not predictive factors for anastomotic leakage or postoperative complications in patients with resected SCLM.
Recently, a diverting stoma has been often used to prevent anastomotic leakage in patients who undergo low anterior resection by diverting the fecal stream and keeping the anastomosis free of material. ${ }^{19,25,26}$ In this study, the presence of a diverting stoma was not a predictive factor for absence of postoperative anastomotic leakage. However, the analysis estimating efficacy of a diverting stoma in this study was not accurate, because a diverting stoma was basically used in patients whose risk for anastomotic leakage was considered to be high by the surgeons. The site of primary tumor that has been reported as a strong predictive factor in previous studies was not a predictive factor for anastomotic leakage in this series. Use of diverting stoma might affect the result of analyses of predictive factors for anastomotic leakage. A randomized, controlled trial is needed to elucidate the efficacy of a temporary diverting stoma.

Although several rationales for the simultaneous resection for SCLM are clear, staged resections should be selected to prevent anastomotic leakage or serious complications when the scheduled operation would result in considerable surgical stress, i.e., predicted operation time greater than $8 \mathrm{~h}$ according to the results of the present study. Predicted operation time should be calculated by considering various factors, such as characteristics of the patient, primary and metastatic tumor, extent of operation, difficulty of the procedure, and so on. Based on the results of this study, we now select staged resections when operation time is expected to be greater than $8 \mathrm{~h}$; otherwise, we select simultaneous resection. A prospective study of SCLM to evaluate the efficacy and safety of the operation time-based decision model is in progress.

Currently, adjuvant chemotherapy is one of the key factors which could affect prognosis. Then, comparison of ratio of patients who could receive adjuvant chemotherapy will be essential when comparing the efficacy of simultaneous resection and that of staged resections in a future study of SCLM. Furthermore, in staged resections, there is a risk that some patients could not undergo a second resection after the first resection due to tumor progression or complication of first surgery. Resection rate of patients who could undergo both primary and hepatic resections 
should be assessed when comparing simultaneous resection and staged resections in SCLM.

The limitations of our study are its retrospective design and the relatively small number of patients studied.

\section{Conclusion}

The morbidity rate and the frequency of anastomotic leakage were high with simultaneous resection for SCLM, especially in patients with greater intraoperative blood loss or operation time greater than $8 \mathrm{~h}$. For patients with SCLM, staged resections should be considered when simultaneous resection would involve excessive surgical stress.

Open Access This article is distributed under the terms of the Creative Commons Attribution Noncommercial License which permits any noncommercial use, distribution, and reproduction in any medium, provided the original author(s) and source are credited.

\section{References}

1. Fong Y, Fortner J, Sun RL, Brennan MF, Blumgart LH. Clinical score for predicting recurrence after hepatic resection for metastatic colorectal cancer: analysis of 1001 consecutive cases. Ann Surg. 1999;230(3):309-318; discussion 18-21.

2. Nordlinger B, Guiguet M, Vaillant JC, Balladur P, Boudjema K, Bachellier P, Jaeck D. Surgical resection of colorectal carcinoma metastases to the liver. A prognostic scoring system to improve case selection, based on 1568 patients. Association Francaise de Chirurgie. Cancer. 1996;77(7):1254-1262.

3. Minagawa M, Makuuchi M, Torzilli G, Takayama T, Kawasaki S, Kosuge T, Yamamoto J, Imamura H. Extension of the frontiers of surgical indications in the treatment of liver metastases from colorectal cancer: long-term results. Ann Surg. 2000;231(4):487-499.

4. Scheele J, Stangl R, Altendorf-Hofmann A, Gall FP. Indicators of prognosis after hepatic resection for colorectal secondaries. Surgery. 1991;110(1):13-29.

5. Capussotti L, Vigano L, Ferrero A, Lo Tesoriere R, Ribero D, Polastri R. Timing of resection of liver metastases synchronous to colorectal tumor: proposal of prognosis-based decisional model. Ann Surg Oncol. 2007;14(3):1143-1150.

6. Chua HK, Sondenaa K, Tsiotos GG, Larson DR, Wolff BG, Nagorney DM. Concurrent vs. staged colectomy and hepatectomy for primary colorectal cancer with synchronous hepatic metastases. Dis Colon Rectum. 2004;47(8):1310-1316.

7. Lyass S, Zamir G, Matot I, Goitein D, Eid A, Jurim O. Combined colon and hepatic resection for synchronous colorectal liver metastases. J Surg Oncol. 2001;78(1):17-21.

8. Martin R, Paty P, Fong Y, Grace A, Cohen A, DeMatteo R, Jarnagin W, Blumgart L. Simultaneous liver and colorectal resections are safe for synchronous colorectal liver metastasis. J Am Coll Surg. 2003;197(2):233-41; discussion 41-42.

9. Fujita S, Akasu T, Moriya Y. Resection of synchronous liver metastases from colorectal cancer. Jpn J Clin Oncol. 2000;30(1):7-11.
10. Lambert LA, Colacchio TA, Barth RJ, Jr. Interval hepatic resection of colorectal metastases improves patient selection. Arch Surg. 2000;135(4):473-9; discussion 9-80.

11. Dindo D, Demartines N, Clavien PA. Classification of surgical complications: a new proposal with evaluation in a cohort of 6336 patients and results of a survey. Ann Surg. 2004;240 (2):205-213

12. Kaplan E. Nonparametric estimation from incomplete observations. J Am Stat Assoc. 1958;53:457-481.

13. Tanaka K, Adam R, Shimada H, Azoulay D, Levi F, Bismuth H. Role of neoadjuvant chemotherapy in the treatment of multiple colorectal metastases to the liver. Br J Surg. 2003;90 (8):963-969.

14. Reddy SK, Pawlik TM, Zorzi D, Gleisner AL, Ribero D, Assumpcao L, Barbas AS, Abdalla EK, Choti MA, Vauthey JN, Ludwig KA, Mantyh CR, Morse MA, Clary BM. Simultaneous resections of colorectal cancer and synchronous liver metastases: a multi-institutional analysis. Ann Surg Oncol. 2007;14(12):34813491.

15. Thelen A, Jonas S, Benckert C, Spinelli A, Lopez-Hanninen E, Rudolph B, Neumann U, Neuhaus P. Simultaneous versus staged liver resection of synchronous liver metastases from colorectal cancer. Int J Colorectal Dis. 2007;22(10):1269-1276.

16. Allen PJ, Kemeny N, Jarnagin W, DeMatteo R, Blumgart L, Fong Y. Importance of response to neoadjuvant chemotherapy in patients undergoing resection of synchronous colorectal liver metastases. J Gastrointest Surg. 2003;7(1):109-115; discussion $16-7$.

17. Mentha G, Majno PE, Andres A, Rubbia-Brandt L, Morel P, Roth AD. Neoadjuvant chemotherapy and resection of advanced synchronous liver metastases before treatment of the colorectal primary. Br J Surg. 2006;93(7):872-878.

18. Verhoef C, van der Pool AE, Nuyttens JJ, Planting AS, Eggermont AM, de Wilt JH. The "liver-first approach" for patients with locally advanced rectal cancer and synchronous liver metastases. Dis Colon Rectum. 2009;52(1):23-30.

19. Karanjia ND, Corder AP, Holdsworth PJ, Heald RJ. Risk of peritonitis and fatal septicaemia and the need to defunction the low anastomosis. Br J Surg. 1991;78(2):196-198.

20. Eberl T, Jagoditsch M, Klingler A, Tschmelitsch J. Risk factors for anastomotic leakage after resection for rectal cancer. Am J Surg. 2008;196(4):592-598.

21. Jung SH, Yu CS, Choi PW, Kim DD, Park IJ, Kim HC, Kim JC. Risk factors and oncologic impact of anastomotic leakage after rectal cancer surgery. Dis Colon Rectum. 2008;51 (6):902-908.

22. Kruschewski M, Rieger H, Pohlen U, Hotz HG, Buhr HJ. Risk factors for clinical anastomotic leakage and postoperative mortality in elective surgery for rectal cancer. Int J Colorectal Dis. 2007;22(8):919-927.

23. Lee WS, Yun SH, Roh YN, Yun HR, Lee WY, Cho YB, Chun HK. Risk factors and clinical outcome for anastomotic leakage after total mesorectal excision for rectal cancer. World J Surg. 2008;32(6):1124-1129.

24. Rullier E, Laurent C, Garrelon JL, Michel P, Saric J, Parneix M. Risk factors for anastomotic leakage after resection of rectal cancer. Br J Surg. 1998;85(3):355-358.

25. Meleagros L, Varty PP, Delrio P, Boulos PB. Influence of temporary faecal diversion on long-term survival after curative surgery for colorectal cancer. Br J Surg. 1995;82(1):21-25.

26. Montedori A, Cirocchi R, Farinella E, Sciannameo F, Abraha I Covering ileo- or colostomy in anterior resection for rectal carcinoma. Cochrane Database Syst Rev. 2010(5):CD006878. 